\section{Transform alfalfa research}

Nat. Commun. 11, 2494 (2020).

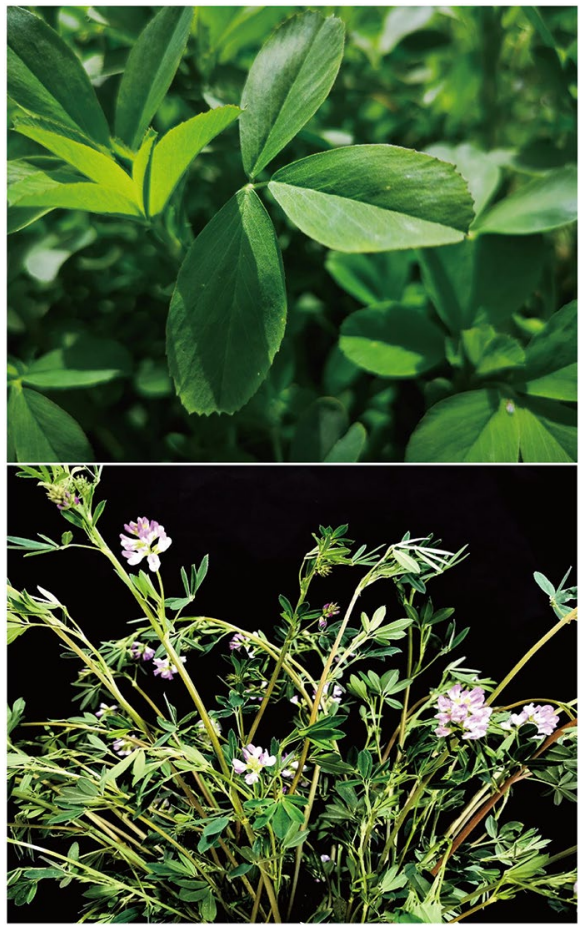

Credit: Guangdong Sanjie Forage Biotechnology Co., Ltd.

Genomic technologies, such as high-throughput sequencing and genome editing, have been rapidly revolutionizing crop research. However, compared to food crops, the important forage crop, cultivated alfalfa (Medicago sativa L.), received fewer benefits from these technologies, largely due to the complex nature of its autotetraploid genome. Previous research on alfalfa has depended on the genome of its sister species, the diploid $M$. truncatula, which does not often serve as a perfect reference. The lack of a reference sequence has been a bottleneck restricting the research and breeding of cultivated alfalfa. To overcome this limitation, Haitao Chen, from Kunming Institute of Zoology and Guangdong Sanjie Forage Biotechnology Co., Ltd., and colleagues developed a chromosome-level genome assembly as well as an efficient genome editing protocol for alfalfa.
The researchers used PacBio circular consensus sequencing, Illumina short-read sequencing and high-throughput chromosome conformation capture technology to assemble this genome. Their assembly pipeline, combining the Canu software package and the ALLHiC algorithm, generated an allele-aware chromosome-level assembly of 2.738 $\mathrm{Gb}$, consisting of 32 super-scaffolds and unplaced contigs. A total of 164,632 protein-coding genes were annotated.

Allelic chromosome pairs showed little sequence divergence or allelic expression dominance, consistent with the features of tetrasomic inheritance. Like other plant species, whole genome duplication and bursts of transposable elements shape the evolution and contribute to the expansion of this genome.

Based on the genome, the researchers constructed a vector containing a CRISPR-Cas9 expression cassette that targeted the Phytoene desaturase gene and transformed alfalfa cultivars using an agrobacteria-mediated approach. Mutant plants, including null mutants displaying dwarf and albino phenotypes, were regenerated and screened based on a selectable marker.

A second gene, PALMATE-LIKE PENTAFOLIATA1 (PALM1), was edited using the same approach. palm1 mutant plants that show palmate-like pentafoliate leaves were generated, providing the possibility to rapidly breed varieties with more leaflets and, consequently, higher biomass and nutritional value. This phenotype showed stable inheritance, and the transfer DNA fragment can be segregated out in $\mathrm{T} 1$ progenies, resulting in transgene-free-edited plants.

The chromosome-level reference genome combined with the efficient genome editing protocol will accelerate the breeding of cultivated alfalfa.

Jun Lyu

Published online: 3 June 2020

https://doi.org/10.1038/s41477-020-0711-6 